\title{
ESTIMATIVA DA IDADE DENTAL PELO MÉTODO DE NICODEMO EM UMA POPULAÇÃO DA REGIÃO SUDESTE DO BRASIL
}

\author{
Sandra França Ribeiro Gobbo \\ Universidade Cidade de São Paulo (UNICID), São Paulo, SP, Brasil \\ Maria Beatriz Carrazzone Cal Alonso \\ União das Faculdades dos Grandes Lagos (UNILAGO), São José do Rio Preto, SP, Brasil \\ Karen Koharu Motooka Kawamoto \\ Universidade Cidade de São Paulo (UNICID), São Paul, SP, Brasil \\ Daniel de Bortoli Teixeira \\ Universidade de Marilia (UNIMAR), Marilia, SP, Brasil \\ Ricardo Henrique Alves da Silva \\ Universidade de São Paulo (FORP-USP), Ribeirão Preto, SP, Brasil \\ Livia Picchi Comar* \\ Universidade de Marilia (UNIMAR), Marilia, SP, Brasil
}

\section{DENTAL AGE ESTIMATION BY NICODEMO METHOD IN A SOUTHEASTERN BRAZILIAN POPULATION}

\section{RESUMO}

Os métodos de estimativa de idade, baseados nos estágios de mineralização dental, são altamente confiáveis e amplamente utilizados para avaliar a idade biológica dos indivíduos. A sua importância se dá principalmente no âmbito forense, desempenhando papel fundamental em questões éticas e legais. Mesmo com a preconização dos vários métodos, ainda permanecem controvérsias quanto à sua aplicação em populações específicas. Portanto, o objetivo deste trabalho foi avaliar a acurácia e aplicabilidade do método de estimativa de idade dental preconizado por Nicodemo em uma população da região sudeste do Brasil. Para isso foi analisado um total de 330 radiografias de tomadas panorâmicas das faces de indivíduos entre seis e dezesseis anos de idade, de ambos os sexos $(\mathrm{n}=30)$. A análise das imagens foi realizada por dois examinadores calibrados ( $\mathrm{CCl} \geq 0,70 ; 95 \%$ IC). Os valores das idades estimadas foram comparados com os valores da idade cronológica da amostra, e então foi contabilizada a porcentagem de acerto do método, dentro de cada grupo etário para ambos os sexos. 0 teste do Qui-quadrado de Pearson foi aplicado para avaliar a influência do sexo. As maiores porcentagens de acerto do método foram encontradas para os grupos de sete a onze anos $(70-90 \%)$, seguidos dos grupos de seis a doze anos (16,7\% e 26,7\%, respectivamente). Já para os grupos de treze a dezesseis anos, nenhum acerto foi observado. 0 método apresentou porcentagens de subestimativa de 15,6 \% (2,1 anos, grupo 13) até 24,3\% (4,0 anos, grupo 16), quando se observou um acréscimo do padrão de erro da técnica conforme 0 aumento da idade cronológica do indivíduo. Conclui-se que, na população avaliada do presente estudo, o método de Nicodemo mostrou-se eficaz para estimar idade nos indivíduos de seis a doze anos, com maior acurácia nos indivíduos de sete a onze anos de idade, sem diferença entre os sexos. Porém, foi ineficaz para estimar idade nos indivíduos de treze a dezesseis anos. Para uma adequada validação e aplicabilidade deste método para esta população específica, mais estudos devem ser realizados.

PALAVRAS-CHAVE: Determinação da idade pelos dentes. Odontologia Legal. Radiologia. Radiografia panorâmica.

\section{ABSTRACT}

Age estimation methods, based on the stages of dental mineralization, are highly reliable and widely used to estimate the biological age of individuals. Its importance occurs mainly in the forensic sphere, playing a fundamental role in ethical and legal issues. Even with the recommendation of these methods, controversies remain regarding the application of the protocols in specific populations. Therefore, the aim of this study was to evaluate the accuracy and applicability of Nicodemo's dental age estimation method in a southeastern Brazilian population. For this, a 
total of 330 panoramic radiographs of individuals between 6 and 16 years of age, of both sexes $(n=30)$ were analyzed. Image analysis was performed by two calibrated examiners (ICC $\geq 0.70 ; 95 \% \mathrm{Cl}$ ). The estimated age values were compared with the real age values of the sample, and then the percentage of correctness of the method was performed, within each age group for both sexes. Pearson's Chi-square test (R Core Team software, 2017) was applied to assess the sex influence. The highest percentages of correctness of the method were found for the groups from 7 to 11 years old (70 - 90\%), followed by the groups of 6 and 12 years old (16.7\% and 26.7\%, respectively). However, for groups between 13 and 16 years of age, no success was observed. The method presented underestimation percentages from $15.6 \%$ (2.1 years, group 13) to 24.3\% (4.0 years, group 16), with an increasing pattern to the error of the technique as the individual's real age increases. It is concluded that, in the population evaluated in the study, the Nicodemo's method proved to be effective in estimating age in individuals aged 6 to 12 years, with greater accuracy in individuals aged 7 to 11 years, with no difference between genders. However, it proved to be ineffective in estimating the age in 13 to 16 years individuals. For an adequate validation and applicability of the method in this specific population, more studies still need to be performed.

\section{KEYWORDS: Age Determination by Teeth. Forensic Dentistry. Radiography. Panoramic.}

\section{INTRODUÇÃO}

A mensuração da idade de um indivíduo é realizada quando a análise do seu desenvolvimento orgânico e dos seus estágios de vida torna-se importante por um motivo específico. A relação entre desenvolvimento e crescimento pode ser expressa como idade cronológica, sendo que os principais índices de maturidade são avaliados por meio da análise das estruturas dentais e ósseas ${ }^{1}$.

Em algumas situações de caráter forense, como identificação de ossadas ou corpos em decomposição, vítimas sem idade comprovada, ou situações de âmbito civil como processos de adoção de menores de idade, identificação de menores infratores, fins de direito (aposentadoria, matrimônio), a estimativa da idade de um indivíduo apresenta grande relevância². Portanto, métodos afins para estimar a idade de indivíduos são de grande aplicabilidade para as ciências forenses, principalmente na rotina de trabalho dos institutos médico-legais (IML's).

A Odontologia Legal, por exemplo, desempenha um papel fundamental em questões éticas e legais, sendo de grande relevância a estimativa da idade cronológica com objetivo de identificação de indivíduos vivos ou mortos, utilizando métodos diretos baseados em exames intraorais, como também métodos indiretos por meio de exames por imagens quando podem ser analisadas as etapas de mineralização dos dentes ${ }^{3,4}$. Sendo assim, essas técnicas radiográficas são extremamente úteis para estimativa de idade e identificação humana, considerando que, na maioria das vezes, as técnicas de análise de DNA não estão disponíveis ${ }^{5}$.

O desenvolvimento dos dentes é considerado como uma sequência de eventos reconhecidamente descritos. Portanto, a sua mineralização e erupção são fatores confiáveis para se estimar a idade de um indivíduo ${ }^{6}$. Nos casos de investigação forense, os dentes têm mostrado um papel fundamental, já que são na- turalmente preservados, mesmo após a desintegração de tecidos e ossos ${ }^{7}$, além da sua especificidade e unicidade, ou seja, cada indivíduo possui dentes com características morfológicas únicas ${ }^{8}$.

Dentre os vários protocolos existentes para o cálculo da estimativa de idade de um indivíduo, os que se baseiam nos estágios de mineralização dental são mais confiáveis quando comparados com os que utilizam o desenvolvimento ósseo, pois a formação dos dentes sofre menos interferência de alterações hormonais, nutricionais e patológicas sistêmicas ${ }^{10,11}$. Alguns autores consideram o método radiográfico para estimativa da idade nessa etapa de desenvolvimento dos dentes como um dos mais seguros e fidedignos para atribuição da idade cronológica uma vez que a radiografia odontológica é uma técnica não destrutiva, além do fato da não influência de fatores externos na formação dos dentes, como já elencado?.

Dentre os métodos utilizados para estimar a idade cronológica de crianças e adolescentes, nos quais os estágios da mineralização dental são avaliados, os mais utilizados são os preconizados por Nolla $(1960)^{12}$, Moorrees et al. $(1963)^{13}$, Demirjian et al. $(1973)^{14} \mathrm{e}$ Willems et al. $(2001)^{15}$. Outra metodologia muito utilizada no Brasil é a prescrita por Nicodemo et al. $(1974)^{16}$, que se baseia nos dez estágios de mineralização de Nolla. Porém, nesta avaliação considera-se apenas oito estágios englobando todos os dentes permanentes, incluindo os terceiros molares, considerando, também, as variações entre os arcos superior e inferior $^{17}$. Os autores desenvolveram uma tabela relacionando a caracterização do estágio de mineralização para cada grupo dentário com a idade cronológica descrita em meses de vida extrauterina, referenciando os valores de Nolla em idades mínima e máxima. Esses parâmetros foram aplicados em uma população leucoderma no estado de São Paulo ${ }^{18}$. No Brasil, as tabelas de Nicodemo são as mais aplicadas, já que os cirurgiões dentistas não necessitam de experiência prévia com o método, que é de fácil aplicabilidade ${ }^{19}$. 
Vários estudos já confirmaram que há divergências nos estágios de mineralização dos dentes quando populações com características raciais diferentes são comparadas ${ }^{4,5,18-22}$. A maturidade dos dentes pode ser influenciada por uma série de fatores que devem ser levados em consideração durante a aplicação de alguns métodos de estimativa de idade. Diferenças culturais e étnicas entre as populações podem explicar as discrepâncias observadas na avaliação da idade dental e isto faz com que novos escores e critérios de classificação sejam determinados para populações específicas ${ }^{23}$. Deste modo, faz-se necessária a avaliação dos métodos já descritos na literatura, considerandose diferentes populações e faixas etárias, com diferentes etnias e culturas.

\section{OBJETIVOS}

O objetivo deste estudo foi avaliar a acurácia e aplicabilidade do método preconizado por Nicodemo para estimativa da idade, baseados na análise da mineralização e erupção dental, em uma população de crianças e adolescentes com idade entre seis a dezesseis anos, residentes no Estado de São Paulo - Brasil.

\section{MATERIAL E MÉTODOS}

\section{Aspectos éticos e legais}

O presente estudo foi aprovado pelo Comitê de Ética em Pesquisa (CEP) em seres humanos da Universidade Cidade de São Paulo-UNICID (CAAE: 69505017.8.0000.0064).

\section{Delineamento experimental e caracterização da amostra}

Este trabalho consistiu de um estudo observacional transversal e cego, em que foram analisadas radiografias panorâmicas digitais de crianças e adolescentes com idade cronológica entre seis a dezesseis anos, de ambos os sexos, do Estado de São Paulo - Brasil. As imagens foram adquiridas do acervo de imagens da empresa "DVI Radiologia Odontológica", Ribeirão Preto - SP, fornecidas por meio da devida assinatura do termo de consentimento livre e esclarecido pela empresa de Radiologia e a devida assinatura do termo de sigilo pelos pesquisadores.

O número amostral utilizado foi de 30 imagens para cada idade $(n=30)$, de ambos os sexos ( $15 \mathrm{M} \mathrm{e} 15 \mathrm{~F}$ ), dando um total de 330 radiografias panorâmicas. As imagens foram selecionadas de acordo com os seguintes critérios de inclusão: radiografias panorâmicas digitais de meninos e meninas entre seis a dezesseis anos de idade, da região sudeste do Brasil, realizadas entre os anos de 2012 a 2017. Os critérios de exclusão foram imagens com baixa nitidez; baixa qualidade e/ou distorções; imagens sem o devido registro da idade cronológica ou sexo do paciente; imagens com presença de agenesias ou extrações dentárias, trauma de face ou qualquer outro tipo de anomalia ou síndromes do desenvolvimento dentário que o paciente porventura apresentasse.

Calibração dos examinadores e avaliação das imagens radiográficas

Todas as radiografias foram analisadas por dois examinadores previamente calibrados, em ambiente escurecido, com auxílio de monitor de 14 polegadas, por meio de um programa específico para visualização de imagens do Windows ${ }^{\circledR}$ (Microsoft Office 2010, Windows ${ }^{\circledR} 8$ ).

A calibração inter e intra-examinador foi apurada mediante cálculo do Coeficiente de Correlação Intraclasse (CCl), através do Software "IBM SPSS (Statistical Package for the Social Sciences) Statistics v22 x 64, para Windows $® "$, modelo de confiabilidade Alfa de Cronbach, com intervalo de confiança de $95 \%$. A calibração foi realizada por meio da análise de onze radiografias panorâmicas que não estavam incluídas no número amostral do estudo. Cada examinador avaliou a mesma radiografia por três vezes, com intervalo de 48 horas entre cada avaliação, pela aplicação do método de Nicodemo. Para a análise intra-examinador foram comparadas as idades dentais estimadas em cada uma das três avaliações de cada examinador. Para a análise inter -examinadores, as idades dentais estimadas pelos dois examinadores foram comparadas entre si.

\section{Aplicação do método de Nicodemo para a estimativa da idade dental \\ Nicodemo et al. $(1974)^{16}$ perceberam que as tabelas es-} trangeiras descritas na literatura não eram compatíveis com a amostra brasileira e vislumbraram a necessidade da avaliação da idade dental a partir de uma tabela com padrões nacionais. Portanto, se basearam nos estágios de mineralização e erupção dentais preconizados por Nolla $(1960)^{12}$ e desenvolveram uma tabela contendo oito estágios de mineralização englobando todos os dentes permanentes, inclusive terceiros molares, e com diferenciação das arcadas superior e inferior.

Este método classifica 0 desenvolvimento dentário em estágios de mineralização de um a oito, desde 0 aparecimento dos primeiros pontos de calcificação até o fechamento do ápice radicular. Estes estágios foram classificados como: 1- início da calcificação da coroa; 2- 1/3 da coroa completa; 3- 2/3 da coroa completa; 4- coroa completa; 5 - início da calcificação da raiz; 61/3 da raiz completa; 7- 2/3 da raiz completa; 8- término apical radicular completo (Figura 1). Com base nos oito estágios de mineralização dental, os autores propuseram a idade estimada em meses (Figura 2). 


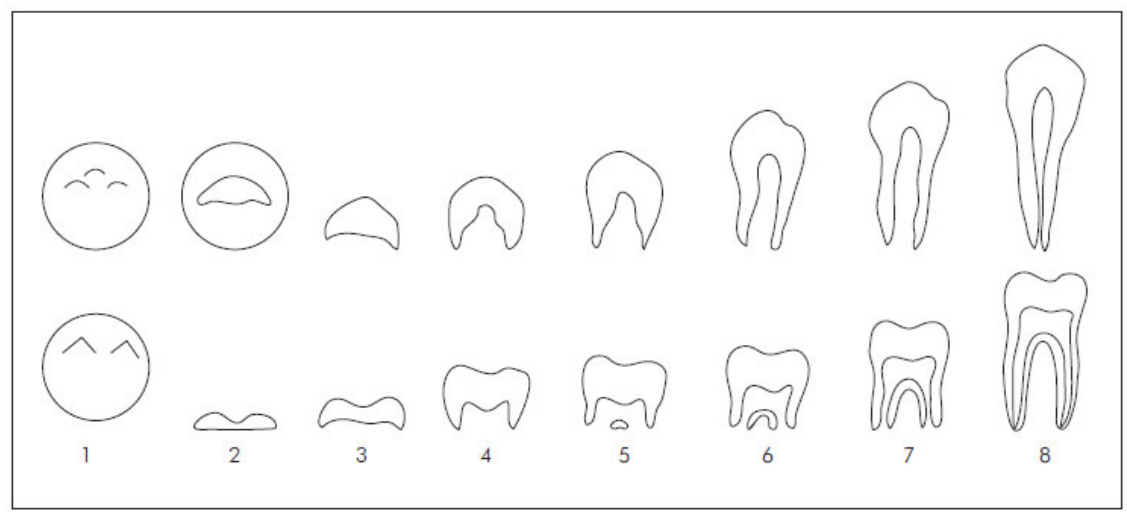

Figura 1: Estágios de mineralização dental propostos por Nicodemo et al. $(1974)^{16}$. Adaptado de Moraes et al. $(2007)^{27}$.

\begin{tabular}{lcccccccc}
\hline \multicolumn{1}{c}{ Dente } & $\begin{array}{c}\text { Evidência } \\
\text { de mineralização }\end{array}$ & $1 / 3$ de coroa & $2 / 3$ de coroa & $\begin{array}{c}\text { Coroa } \\
\text { completa }\end{array}$ & $\begin{array}{c}\text { Início da } \\
\text { formação } \\
\text { radicular }\end{array}$ & $1 / 3$ da raiz & $2 / 3$ da raiz & $\begin{array}{c}\text { Término } \\
\text { apical }\end{array}$ \\
\hline Superiores & & & & & & & \\
Incisivo central & $5-7$ & $8-15$ & $18-30$ & $36-57$ & $60-78$ & $75-90$ & $87-108$ & $100-116$ \\
Incisivo lateral & $9-15$ & $24-30$ & $33-57$ & $54-72$ & $72-88$ & $84-102$ & $96-112$ & $105-117$ \\
Canino & $5-6$ & $12-33$ & $36-60$ & $60-78$ & $76-87$ & $90-114$ & $111-141$ & $126-156$ \\
$1^{\circ}$ pré-molar & $27-30$ & $48-66$ & $57-75$ & $78-96$ & $87-108$ & $102-126$ & $117-138$ & $129-159$ \\
$2^{\circ}$ pré-molar & $36-54$ & $51-66$ & $66-84$ & $78-102$ & $93-117$ & $105-129$ & $117-144$ & $141-159$ \\
$1^{\circ}$ molar & $1-6$ & $6-16$ & $18-30$ & $36-48$ & $54-66$ & $66-84$ & $75-96$ & $90-104$ \\
$2^{\circ}$ molar & $39-57$ & $52-66$ & $69-84$ & $81-102$ & $102-126$ & $120-134$ & $129-153$ & $150-162$ \\
$3^{\circ}$ molar & $90-132$ & $96-138$ & $102-156$ & $138-174$ & $162-198$ & $182-208$ & $185-238$ & $216-245$ \\
Inferiores & & & & & & & & \\
Incisivo central & $3,9-6,1$ & $9-12$ & $18-27$ & $28-45$ & $48-68$ & $60-78$ & $76-96$ & $90-102$ \\
Incisivo lateral & $4,6-5,8$ & $7-12$ & $18-30$ & $18-66$ & $54-78$ & $68-88$ & $80-99$ & $92-102$ \\
& & & & & & & & \\
Canino & $4-7$ & $8-30$ & $24-54$ & $51-72$ & $69-93$ & $84-108$ & $105-135$ & $129-156$ \\
$1^{\circ}$ pré-molar & $27-36$ & $45-60$ & $51-72$ & $69-90$ & $84-102$ & $102-126$ & $114-141$ & $132-156$ \\
$2^{\circ}$ pré-molar & $33-54$ & $48-63$ & $66-81$ & $78-96$ & $93-144$ & $108-132$ & $117-144$ & $141-159$ \\
$1^{\circ}$ molar & $1-6$ & $6-12$ & $18-28$ & $18-45$ & $54-66$ & $57-81$ & $78-96$ & $90-104$ \\
$2^{\circ}$ molar & $39-60$ & $51-66$ & $72-87$ & $84-105$ & $102-126$ & $117-135$ & $129-153$ & $150-165$ \\
$3^{\circ}$ molar & $90-132$ & $96-138$ & $102-156$ & $138-174$ & $162-198$ & $182-208$ & $185-238$ & $216-245$ \\
\hline
\end{tabular}

Figura 2: Tabela cronológica da mineralização dos dentes permanentes entre brasileiros proposta por Nicodemo et al. (1974) ${ }^{16}$. Adaptado de Miranda et al. $(2015)^{22}$. *Idade estimada em meses de vida.

Para a análise das amostras, as radiografias panorâmicas foram mascaradas (cegamento da avaliação), de forma que os examinadores não permanecessem tendenciosos durante as avaliações. Assim, todas as informações referentes aos pacientes que estavam contidas nas imagens radiográficas foram inicialmente ocultadas, impossibilitando a identificação do indivíduo. Os arquivos das imagens digitais foram identificados por números e apenas quando as avaliações finalizaram, os examinadores puderam ter acesso à identificação dos indivíduos.

A análise foi realizada com o auxílio de fichas de avaliação, sendo a amostra total no valor de, no máximo, vinte imagens por dia a fim de se evitar a fadiga visual e, consequentemente, 0 comprometimento das avaliações.

Foram analisadas 330 radiografias panorâmicas digitais de uma população de crianças e adolescentes do estado de São
Paulo, entre seis a dezesseis anos de idade. As imagens foram divididas em onze grupos de análise, de acordo com a idade cronológica dos indivíduos, sendo que metade da amostra pertencia ao sexo masculino (M) e a outra metade ao sexo feminino (F), considerando um número amostral de 30 imagens por grupo etário ( $n=30,15 \mathrm{M}$ e $15 \mathrm{~F}, 11$ grupos de idade).

As radiografias panorâmicas foram analisadas com base nos estágios de mineralização dos dentes permanentes inferiores esquerdos em cada imagem radiográfica selecionada, de acordo com os estágios de calcificação preconizados no método. Com 0 auxílio de fichas de avaliação, os examinadores identificaram os estágios nos dentes $34,35,36,37$ e 38 e, em seguida, a idade estimada do indivíduo foi calculada através da idade mínima e máxima para cada dente avaliado de acordo com a tabela de mineralização proposta pelo método (Figura 3). 


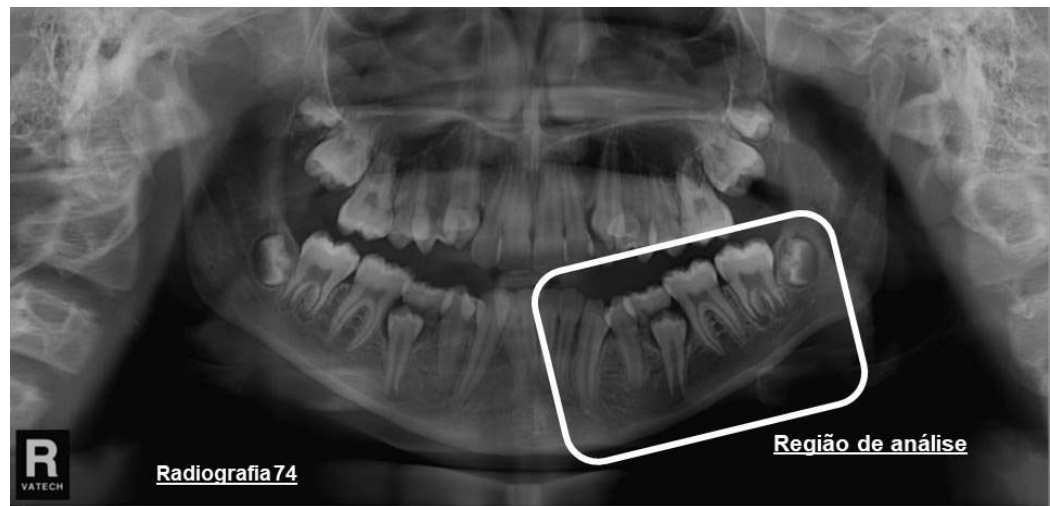

\begin{tabular}{|c|c|c|c|}
\hline \multicolumn{4}{|c|}{ Radiografia 74 Feminino } \\
\hline Dente & Estágio & $\begin{array}{c}\text { Idade minima } \\
\text { (meses) }\end{array}$ & $\begin{array}{c}\text { Idade máxima } \\
\text { (meses) }\end{array}$ \\
\hline $34\left(1^{\circ}\right.$ pré-molar $)$ & 8 & 132 & 156 \\
\hline $35\left(2^{\circ}\right.$ pré-molar $)$ & 7 & 117 & 144 \\
\hline $36\left(1^{\circ}\right.$ molar $)$ & 8 & 90 & 104 \\
\hline $37\left(2^{\circ}\right.$ molar $)$ & 7 & 129 & 153 \\
\hline $38\left(3^{\circ}\right.$ molar $)$ & 4 & 138 & 174 \\
\hline \multicolumn{2}{|c|}{ Média } & \multicolumn{6}{|c|}{ Intervalo médio da idade estimada: 10,1 a 12,1 anos } \\
\hline
\end{tabular}

Figura 3: Figura ilustrativa exemplificando a esquematização das fichas de análise das radiografias panorâmicas pelo método de Nicodemo.

Os seguintes passos foram fielmente seguidos durante a análise das imagens:

- comparou-se a imagem radiográfica de cada um dos dentes referidos com os estágios de mineralização propostos pelo método;

- estimou-se o estágio de mineralização de cada dente analisado e localizou-se o intervalo de tempo (em meses) correspondente, sendo anotadas as idades mínima e máxima estimadas (em meses) para cada dente;

- obteve-se a média da idade mínima e a média da idade máxima estimada de todos os dentes avaliados, convertida em anos, ao final da análise de todos os dentes;

- encontrou-se o intervalo médio de idade estimada do indivíduo;

- considerou-se o dente homólogo (lado direito inferior) nos casos de dentes ausentes no lado esquerdo inferior;

- considerou-se, também, o dente homólogo nos casos com dificuldades de avaliação, por falta de nitidez da imagem, em um dente específico;

- comparou-se 0 intervalo médio da idade estimada de cada indivíduo com a sua idade cronológica real, após as análises radiográficas que, até então, era desconhecida pelo pesquisador.

Todos os valores de estimativa de idade obtidos foram tabulados em planilhas do Excel e comparados com a idade cronológica real dos indivíduos. Esta avaliação foi realizada por um terceiro pesquisador, o mesmo que realizou o cegamento das imagens.

\section{Análise estatística}

Os valores do intervalo médio da idade estimada foram comparados com os valores da idade real média da população estudada, em cada grupo de idade analisado, sendo contabili- zados os valores referentes à porcentagem de acerto e erro do método, dentro de cada grupo etário para ambos os sexos. Os dados foram analisados quanto à normalidade e homogeneidade (teste de Kolmogorov-Smirnov, $p<0,05$ ) e tendo sido encontrados valores não homogêneos em relação à idade, o teste do Qui-quadrado de Pearson (software R Core Team, 2017) foi utilizado para avaliar a influência do sexo, na estimativa de idade encontrada dentro de cada grupo de idade analisado. 0 nível de significância adotado foi de $5 \%$.

\section{RESULTADOS}

Para a calibração dos avaliadores, foi obtido um $\mathrm{CCl}$ intra -examinador de 0,846 para o examinador 1 e um $\mathrm{CCl}$ intra-examinador de 0,997 para o examinador 2, e um CCl inter-examinador de 0,884 . Como um $\mathrm{CCl} \geq 0,70$ (IC de $95 \%$ ) é considerado aceitável, os coeficientes intraclasse obtidos no presente estudo para o método de Nicodemo foram considerados dentro do intervalo aceitável para a confiabilidade da metodologia aplicada.

Foram analisadas 330 radiografias panorâmicas digitais de uma população de crianças e adolescentes do Estado de São Paulo, entre seis a dezesseis anos de idade. As imagens foram divididas em onze grupos de análise, de acordo com a idade cronológica dos indivíduos, sendo que metade da amostra pertencia ao sexo masculino e a outra metade ao sexo feminino, considerando um número amostral de 30 imagens por grupo etário de análise ( $n=30,15 \mathrm{M} \mathrm{e} 15 \mathrm{~F}, 11$ grupos de idade). Para cada faixa etária estudada, inicialmente foi realizada a análise do intervalo médio da idade estimada (média da idade mínima e média da idade máxima estimada), pela aplicação do método e, então, o valor do intervalo médio estimado foi comparado com o valor da média da idade real da população, obtendo-se, assim, o valor da porcentagem de acertos entre as idades (Tabela 1). 
Tabela 1: Porcentagem de acerto do intervalo médio da idade estimada em comparação à média da idade real da população estudada, para cada grupo etário analisado pelo método de Nicodemo ( $n=30,15 \mathrm{M} \mathrm{e} 15 \mathrm{~F}$ ).

\begin{tabular}{ccccc}
\hline $\begin{array}{c}\text { Grupos de } \\
\text { análise }\end{array}$ & $\begin{array}{c}\text { Média da idade } \\
\text { estimada mínima } \\
\text { (anos, } \pm \text { d.p.) }\end{array}$ & $\begin{array}{c}\text { Média da idade } \\
\text { estimada máxima } \\
\text { (anos, } \pm \text { d.p.) }\end{array}$ & $\begin{array}{c}\text { Média da } \\
\text { idade real } \\
\text { (anos, } \pm \text { d.p.) }\end{array}$ & $\begin{array}{c}\text { \% acerto * } \\
\text { (n= 30) }\end{array}$ \\
\hline 6 anos & $6,7( \pm 0,4)$ & $8,6( \pm 0,7)$ & $6,5( \pm 0,3)$ & $16,67 \%$ \\
7 anos & $7,1( \pm 0,5)$ & $9,1( \pm 0,8)$ & $7,4( \pm 0,3)$ & $76,67 \%$ \\
8 anos & $7,9( \pm 0,6)$ & $10,0( \pm 0,6)$ & $8,4( \pm 0,3)$ & $83,33 \%$ \\
9 anos & $8,6( \pm 0,8)$ & $10,8( \pm 0,8)$ & $9,4( \pm 0,3)$ & $76,67 \%$ \\
10 anos & $9,3( \pm 0,7)$ & $11,4( \pm 0,6)$ & $10,5( \pm 0,3)$ & $90,00 \%$ \\
11 anos & $9,5( \pm 0,7)$ & $11,6( \pm 0,6)$ & $11,5( \pm 0,3)$ & $70,00 \%$ \\
12 anos & $10,2( \pm 0,6)$ & $12,2( \pm 0,5)$ & $12,5( \pm 0,3)$ & $26,67 \%$ \\
13 anos & $10,4( \pm 0,5)$ & $12,4( \pm 0,4)$ & $13,5( \pm 0,3)$ & $0,00 \%$ \\
14 anos & $11,0( \pm 0,6)$ & $12,9( \pm 0,5)$ & $14,5( \pm 0,3)$ & $0,00 \%$ \\
15 anos & $11,3( \pm 0,5)$ & $13,2( \pm 0,5)$ & $15,4( \pm 0,3)$ & $0,00 \%$ \\
16 anos & $11,5( \pm 0,4)$ & $13,4( \pm 0,5)$ & $16,4( \pm 0,3)$ & $0,00 \%$ \\
\hline *Dados descritivos de correlação simples (porcentagem de acerto, $\mathrm{n}=30$ por grupo, $15 \mathrm{M} \mathrm{e} 15 \mathrm{~F})$.
\end{tabular}

Os maiores valores percentuais de acertos da comparação do intervalo médio da idade estimada com a idade real foram observadas nos grupos de sete a onze anos de idade, com as respectivas porcentagens de acertos da idade estimada: 76,7\%; $83,3 \% ; 76,7 \%$; $90,0 \%$ e $70,0 \%$, seguidos pelos grupos de seis a doze anos de idade, em que foram observados menores porcentagens de acertos: $16,7 \%$ e $26,7 \%$, respectivamente. Para os grupos de treze a dezesseis anos de idade, não houve percentual de acerto da idade estimada pelo método de Nicodemo em comparação à idade real da população.

Os resultados obtidos passaram também pelo teste de Qui-quadrado de Pearson, com o objetivo de avaliar se o sexo interferiu na estimativa de idade encontrada em cada grupo etário. Foi observado que, tanto de uma forma geral como de forma específica para cada faixa etária, para todos os grupos não houve diferença estatística significante entre os sexos para a estimativa da idade dental (porcentagem de acerto da idade estimada; Tabela 2).

Tabela 2: Porcentagem de acerto do intervalo médio da idade estimada em comparação à média da idade real da população estudada, para cada sexo, referente a cada grupo etário analisado pelo método de Nicodemo ( $\mathrm{n}=15$ para ambos os sexos).

\begin{tabular}{cccc}
\hline Grupos de análise & $\begin{array}{c}\% \text { acerto } \\
\text { sexo masculino }\end{array}$ & $\begin{array}{c}\% \text { acerto } \\
\text { sexo feminino }\end{array}$ & Valor de $\mathbf{p}^{*}$ \\
\hline 6 anos & $20,00 \%$ & $13,33 \%$ & $\mathrm{p}=0,624$ \\
7 anos & $73,33 \%$ & $80,00 \%$ & $\mathrm{p}=1,000$ \\
8 anos & $80,00 \%$ & $86,67 \%$ & $\mathrm{p}=1,000$ \\
9 anos & $73,33 \%$ & $80,00 \%$ & $\mathrm{p}=1,000$ \\
10 anos & $93,33 \%$ & $86,67 \%$ & $\mathrm{p}=1,000$ \\
11 anos & $53,33 \%$ & $86,67 \%$ & $\mathrm{p}=0,109$ \\
12 anos & $33,33 \%$ & $20,00 \%$ & $\mathrm{p}=0,682$ \\
\hline
\end{tabular}

* Teste do Qui-quadrado de Pearson: correlação entre o sexo e a porcentagem de acertos para cada grupo etário, $\mathrm{p}<0,05$. Os grupos de 13 a 16 anos não foram apresentados, pois apresentaram $0 \%$ de acerto da idade estimada em relação à idade real.

Em geral, para os grupos em que houve pouca ou nenhuma porcentagem de acerto do método em comparação à idade real, observou-se que houve uma subestimativa do método, ou seja, o método de Nicodemo subestimou a idade dos indivíduos analisados (Tabela 3). 
Tabela 3: Porcentagem de subestimativa da média da idade estimada em comparação à média da idade real da população estudada, para cada grupo etário analisado pelo método de Nicodemo (n=30, $15 \mathrm{M} \mathrm{e} 15 \mathrm{~F}$ ).

\begin{tabular}{cccc}
\hline $\begin{array}{c}\text { Grupos de } \\
\text { análise* }\end{array}$ & $\begin{array}{c}\text { Média da idade } \\
\text { estimada } \\
\text { (anos, } \pm \text { d.p.) }\end{array}$ & $\begin{array}{c}\text { Média da idade } \\
\text { real } \\
\text { (anos, } \pm \text { d.p.) }\end{array}$ & $\begin{array}{c}\text { \% média } \\
\text { Subestimativa }\end{array}$ \\
\hline 13 anos & $11,4( \pm 1,4)$ & $13,5( \pm 0,3)$ & $15,6 \%(2,1$ anos $)$ \\
14 anos & $11,9( \pm 1,3)$ & $14,5( \pm 0,3)$ & $17,7 \%(2,6$ anos $)$ \\
15 anos & $12,2( \pm 1,3)$ & $15,4( \pm 0,3)$ & $20,9 \%(3,2$ anos $)$ \\
16 anos & $12,4( \pm 1,3)$ & $16,4( \pm 0,3)$ & $24,3 \%(4,0$ anos $)$ \\
*Apenas os grupos que apresentaram $0 \%$ de acerto da idade estimada em relação à idade real \\
estão apresentados.
\end{tabular}

Foi observada uma porcentagem média de subestimativa de $15,6 \%$ (2,1 anos) pra o grupo de treze anos; $17,7 \%(2,6$ anos) para o grupo de quatorze anos; $20,9 \%$ (3,2 anos) para 0 grupo de quinze anos e 24,3\% (4,0 anos) para o grupo de dezesseis anos. Portanto, observou-se um padrão de aumento da subestimativa conforme o aumento da idade real do indivíduo, a partir dos treze anos.

\section{DISCUSSÃO}

No presente estudo, foi avaliada a estimativa da idade dental por meio da aplicação do método de Nicodemo, método em que são observados os estágios de mineralização e erupção dos dentes permanentes. Baseando-se nesses estágios, a idade dental é estimada pela análise dos intervalos de idade obtidos (média da idade mínima e média da idade máxima). Para uma maior facilidade e acurácia do método, foram escolhidos os dentes 34 a 38 para a realização das análises, já que muitos estudos mostraram uma maior acurácia no método de Nicodemo quando apenas quatro a cinco dentes são utilizados na avaliação. Moreno et al. (2014) ${ }^{18}$ avaliaram o índice de acerto da idade cronológica de jovens entre dez a 25 anos de idade, utilizando o método de Nicodemo em uma população paraibana, e observaram maior percentual de acerto quando analisaram apenas quatro dentes por radiografia panorâmica $(81,9 \%)$ em relação à análise de dezesseis dentes $(4,3 \%)$.

É importante salientar que grande parte dos trabalhos que avaliaram a acurácia da aplicação do método de Nicodemo em populações específicas, analisou o intervalo de idade estimada, ou seja, calcularam a média da idade estimada mínima e a média da idade estimada máxima para cada dente analisado e depois calcularam a média da idade estimada mínima e máxima do indivíduo, estipulando, assim, um intervalo de idade cronológica provável do indivíduo ${ }^{18,19,22}$. Foi observado, no presente estudo, uma maior porcentagem de acerto para os grupos de sete a onze anos de idade (variando de 70 a $90 \%$ de acerto), seguidos pelos grupos de seis a doze anos (16 a 27\% de acerto, aproximadamente). Já para os grupos de treze a dezesseis anos, não houve percentual de acerto do método para estimar a idade da popula- ção avaliada.

Muitos trabalhos mostraram diferenças consistentes entre a idade dental estimada pelo método de Nicodemo em relação à idade cronológica dos indivíduos. Essas divergências já foram observadas tanto em resultados que apresentaram subestimativa, quanto em resultados que apresentaram superestimativa em relação à idade real, com variações em torno de seis semanas até dezenove meses ${ }^{22,24-26}$. Portanto, os resultados encontrados neste trabalho estão de acordo com os registrados na literatura, já que se constatou uma diferença de correlação variando de dois a quatro anos, havendo a possibilidade de validação deste método nesta população específica. Porém, o número amostral do presente estudo ainda é considerado pequeno para a validação do método. Desta forma, mais estudos devem ser realizados.

Neste trabalho observou-se que o método de Nicodemo foi eficaz em estimar a idade dental na população de seis a doze anos, sendo mais preciso para os grupos de sete a onze anos, onde foram observadas as maiores porcentagens de acerto, sendo a maior encontrada no grupo de dez anos (90\% de acerto). Já para a população de treze a dezesseis anos, o método mostrouse ineficaz, não apresentando percentual de acertos da idade estimada em comparação à idade real. Com relação ao sexo, não foi encontrada diferença significante entre meninos e meninas para a estimativa da idade, ou seja, o método parece ter a mesma acurácia para estimar idade tanto em indivíduos do sexo masculino quanto do sexo feminino.

Sendo assim, os presentes resultados estão de acordo com resultados da literatura. Oliveira et al. (2010)19 avaliaram a aplicabilidade do método de Nicodemo em uma população da região Centro-Oeste do Brasil. Os autores analisaram 200 radiografias panorâmicas, de ambos os sexos, e verificaram apenas $54 \%$ de acertos da estimativa de idade por este método. Os autores ainda observaram que a partir dos quatorze anos de idade, houve um aumento significativo de erros ao se comparar a idade real com a estimada, e que provavelmente isto seja devido à diminuição do número de dentes que se encontram em estágio de mineralização acima desta idade, ou seja, passíveis de serem analisados por este método. Da mesma forma, Moreno et al.(2014) ${ }^{18}$ avaliaram 0 índice de acerto da idade cronológica 
de jovens entre dez a 25 anos de idade, utilizando o método de Nicodemo em uma população paraibana. Os autores observaram maior percentual de acerto quando analisaram apenas quatro dentes por radiografia panorâmica $(81,9 \%)$ em relação à análise de dezesseis dentes (4,3\%). Além disso, observaram que o percentual de acerto foi maior nos indivíduos entre dez a quinze anos $(94,4 \%)$ em relação aos com mais de quinze anos (65\%). Os autores discutiram que este método dificulta a estimativa da idade de indivíduos entre quinze a dezoito anos que é a faixa etária mais importante dentro dos institutos médico-legais, devido à necessidade de se distinguir menores infratores de adultos. Miranda et al. (2015) 22 afirmaram que métodos que avaliam os intervalos de idade estimada, conseguem um maior número de acertos, porém esclareceram que apesar do método de Nicodemo ser considerado de grande aplicabilidade e de fácil utilização, ainda observam-se dados discrepantes quando da sua utilização, já que o desenvolvimento do indivíduo pode ser influenciado por diversos fatores, havendo diferenças nos estágios de mineralização dos dentes entre indivíduos dos diferentes sexos e etnias. Esta questão também foi observada no presente estudo, em que muitos indivíduos da mesma idade e mesmo sexo apresentaram diferentes estágios de mineralização para o mesmo grupo dentário (dados apenas observacionais), prejudicando a análise da idade estimada pelo método de Nicodemo.

Uma possível explicação pode estar relacionada ao fato de que a população analisada foi heterogênea, pois foram selecionados indivíduos residentes no estado de São Paulo, sem distinção de raça e origem, já que estes dados eram inacessíveis aos pesquisadores, visto que nos documentos disponibilizados pela empresa de radiologia estavam presentes apenas a radiografia panorâmica e as informações da idade e sexo do indivíduo. Este fato poderia explicar a menor porcentagem de acerto nos grupos de idade entre seis a doze anos.

Portanto, mediante a análise dos resultados encontrados, foi observado que o método de Nicodemo de estimativa da idade dental é um método simples e de fácil aplicabilidade, não tendo sido encontradas dificuldades durante a sua aplicação. 0 método apresentou ser eficaz para estimar a idade em indivíduos mais jovens, entre seis a doze anos de idade, com maior acurácia nos indivíduos de sete a onze anos de idade. Para indivíduos de treze a dezesseis anos de idade, o método foi ineficaz. Não foram encontradas diferenças na estimativa de idade entre os sexos para um mesmo grupo de idade. Uma maior busca de dados em relação à estimativa de idade pelo método de Nicodemo nesta população específica ainda deve ser realizado. Os presentes resultados devem ser confirmados em outros estudos observacionais utilizando um maior número amostral, para uma adequada validação e aplicabilidade do método.

\section{CONCLUSÃO}

O método de Nicodemo para estimativa da idade dental, na população avaliada, apresentou ser eficaz em estimar idade em indivíduos entre seis a doze anos de idade, com maior acurácia em indivíduos de sete a onze anos de idade, sem diferença entre os sexos; e ineficaz em estimar idade em indivíduos de treze a dezesseis anos de idade. Para uma adequada validação e aplicabilidade do método nesta população específica, outros estudos ainda devem ser realizados.

\section{AGRADECIMENTOS}

Agradecemos a empresa "DVI Radiologia Odontológica", Ribeirão Preto- SP, a qual gentilmente forneceu as imagens radiográficas para a análise do presente estudo.

\section{REFERÊNCIAS}

1.Marshall D. Radiographic correlation of hand, wrist, and tooth development. Dent Radiogr Photogr. 1976;49(3):51-72.

2.Olze A, Reisinger W, Geserick G, Schmeling A. Age estimation of unaccompanied minors. Part II. Dental aspects. Forensic Sci Int. 2006;15(159):65-67.

3.Bérgamo AL, De Queiroz CL, Sakamoto HE, Silva RHA. Dental Age Estimation Methods in Forensic Dentistry: Literature Review. Peertechz J Forensic Sci Technol. 2016;1:17-22.

4.Tonin LO, Leite NLP, Galo R, Silva RHA. Age estimation based on the stage of mineralization of third molars on orthopantograms. Biosci J. 2016;32(3):805-812.

5.Gundim AC, Sousa AP, Silva JC, De Oliveira R, Yamamoto-Silva FP, Silva BSF. Estágio de mineralização dos terceiros molares e sua relação com a idade cronológica: uma amostra da população do Centro-Oeste do Brasil. Rev Odontol UNESP. 2014;43(5):294-298. 6.Nyström M, Haataja J, Kataja M, Evälahti M, Peck L, Kleemola -Kujala E. Dental maturity in Finnish children, estimated from the development of seven permanent mandibular teeth. Acta Odontol Scand. 1986;44(4):193-198.

7.Bhat VJ, Kamath GP. Age estimation from root development of mandibular third molars in comparison with skeletal age of wrist joint. Am J Forensic Med Pathol. 2007;28(3):238-241.

8.Meinl A, Huber CD, Tangl S, Gruber GM. Teschler-Nicola M, Watzek, G. Comparison of the validity of three dental methods for the estimation of age at death. Forensic Sci Int. 2008;178:96-105.

9.Gruber J, Kameyama MM. O papel da Radiologia em Odontologia Legal. Pesqu Odontol Bras. 2001;15(3):263-268.

10.Silva DD, Crês FM, Lédo JP, Tavano O, Carvalho IMM. Idade biológica de pacientes portadores de fissura lábio-palatal estimada pelos índices de Tanner-Whiterhouse (idade óssea) e Souza-Freitas (idade dentária). Rev ABRO. 2000;1(1):21-26.

11.Cornélio WLN, Conélio GC, Conceição MB. Estimativa da idade pelos $3^{\circ} \mathrm{S}$ molares através de RX: relato de caso. RGO. 2006;54(3):230-233.

12.Nolla CM. The development of permanent teeth. J Dent Child. 
1960;27(4):254-266.

13.Moorrees CFA, Fanning EA, Hunt JREE. Age variation of formation stages for tem permanent teeth. J Dent Res. 1963;42(6):1490-1502. 14.Demirjian A, Goldstein $H$, Tanner JM. A new system of dental age assessment. Hum Biol. 1973;45(2):211-227 .

15.Willems G, Van Olmen A, Spiessens B, Carels C. Dental age estimation in Belgian children: Demirjian's technique revisited. J Forensic Sci. 2001;46(4):893-895.

16.Nicodemo RA, Moraes LC, Médici FE. Tabela cronológica da mineralização dos dentes permanentes entre brasileiros. Rev Fac Odont São José dos Campos. 1974;3(1):55-56.

17.Junior EF, Moura LCL. A importância dos arcos dentários na identificação humana. Rev. Bras. Odontol. do Rio de Janeiro. 2014;71(1):22-27.

18.Moreno MBP, Pontes TJP, Rabello PM. Utilização da tabela de cronologia de mineralização dental de Nicodemo, Moraes e Médici Filho na estimativa da idade de paraibanos. Saúde, Ética \& Justiça. 2014;19(1):35-44.

19. Oliveira OF, Fernandes MM, Daruge Júnior E, Melani RFH, Paranhos $L R$. Estimativa da idade por meio de radiografias panorâmicas. Rev Gaúcha Odontol. 2010;58(2):203-206.

20.Chaillet N, Willems G, Demirjian A. Dental maturity in Belgian children using Demirjian's method and polynomial functions: new standard curves for forensic and clinical use. J Forensic Odontostomatol. 2004;22(2):18-27.

21.De Oliveira FT, Capelozza AL, Lauris JR., De Bullen IR. Minera- lization of mandibular third molars can estimate chronological age-Brazilian indices. Forensic Sci Int. 2012;219(1-3):147-150.

22.Miranda SS, Das Neves DMP, Gomes FJS, Corte-Real AT. Estimativa da idade pela mineralização dentária utilizando o método de Nicodemo, Morais e Médici Filho (1974) em população portuguesa. Arq Odontol. 2015;51(3):158-164.

23.Maia MCG, Martins MGA, Germano FA, Brandão Neto J, Silva $C A B$. Demirjian's system for estimating the dental age of northeastern Brasil chindren. Forensic Sci Int. 2010;200(1-3):177.

24.Silva SRP, Nouer PRA, Garbui IU, Ramalho A.S. Definição da época para início do tratamento ortodôntico: avaliação da idade cronológica e dentária em leucodermas. RGO. 2005;53(4):273-276.

25.Kohatsu LI. Estudo radiográfico da relação entre os principais métodos de avaliação da idade óssea e dentária com a idade cronológica quando aplicados à leucodermas e xantodermas brasileiros. [Tese de Doutorado]. São José dos Campos. Universidade Estadual Paulista, Faculdade de Odontologia de São José dos Campos; 2008.

26. Rossi GN. Estudo comparativo da idade dentária segundo Dermijian e Nicodemo em radiografias panorâmicas de crianças de 6 a 11 anos. [Monografia de Especialização em Radiologia, Imaginologia e Estomatologia Odontológica]. Campinas. Faculdade de Odontologia São Leopoldo Mandic; 2016.

27.Moraes ME, Bastos MS, Santos LR, Castilho JC, Moraes LC, Medici Filho E. Dental age in patients with Down syndrome. Braz Oral Res. 2007;21(3):259-264. 